\title{
Хромогранин А в диагностике феохромоцитомы (сравнительный анализ)
}

\author{
М.Ю. Юкина ${ }^{\bowtie}$, П.А. Карпова, Е.А. Трошина, Н.М. Платонова, А.Г. Бельцевич \\ ФГБУ «Национальный медицинский исследовательский центр эндокринологии» Минздрава России, Москва, Россия
}

\begin{abstract}
Аннотация
Цемь. Изучить прогностическую ценность определения хромогранина А (ХрА) в Аиагностике фреохромоцитомы (ФХЦ).

Материалы и методы. Выполнено сравнительное аналитическое исследование с участием 157 пациентов с подозрением на ФХЦ, провомилась статистическая обработка результатов анализа суточной мочи на метанефрин (МН) и норметанефрин, а также анализа крови на

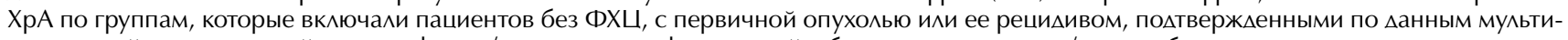
спиральной компьютерной томографии, и/или сцинтиграфии с метайодбензилгуанидином, и/или пробы с кАонидином.

Результаты. Рассчитаны показатели эффрективности мето $\triangle$ ов по группам и отмечено, что наиболее низкие цифры чувствительности метода определения ХрА наблюдаются в группе с рецидивом ФХЦ (43,8\%). При ее исключении из общей выборки специфичность метода сохранилась на высоком уровне (85,45\%), а чувствительность значимо возросла - $А$ 87,1\%. Также значимо повысился и показатель

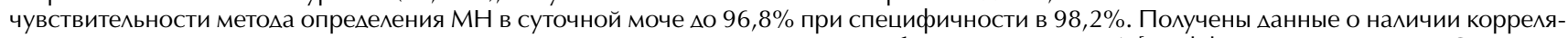
ционной связи между диаметром опухоли и ее секреторной активностью: слабая - с уровнем ХрА [коэфриицент корреляции Спирмена (rho) 0,491] и сильная с суммарным уровнем метилированных катехоламинов - МКА (rho 0,765). ^ожноположительные результаты чаше отмечались у пациентов с Аругими нейроэндокринными опухолями (37,5\%), а также у принимаюших ингибиторы протонной помпы (43,75\%). Чувствительность и специфичность метода определения ХрА в группе пациентов с результатами анализа на МН в пределах «серой зоны» оказались 50 и 86,1\% соответственно.

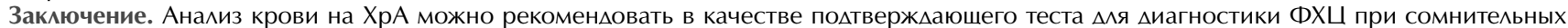
показателях МКА или при подозрении на рецидив ФХЦ. Применение теста в качестве метода 1-го ря аа - только при отсутствии возможности исследования МКА. При интерпретации ХрА необходимо учитывать состояния, вызываюшие ложноположительные результаты.
\end{abstract}

КАючевые слова: фреохромоцитома, хромогранин А, метанефрин, норметанефрин, катехоламины

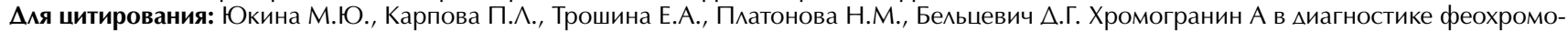
цитомы (сравнительный анализ). Терапевтический архив. 2021; 93 (4): 389-396. DOI: 10.26442/00403660.2021.04.200678

\section{Chromogranin $A$ in diagnosis of pheochromocytoma (comparative analysis)}

Marina Yu. Yukina ${ }^{凶}$, Polina L. Karpova, Ekaterina A. Troshina, Nadezhda M. Platonova, Dmitry G. Beltsevich

Endocrinology Research Centre, Moscow, Russia

\begin{abstract}
Aim. To study the prognostic value of determining Chromogranin A blood level in the diagnosis of PHEO.

Materials and methods. We conducted a comparative analytical study of 157 patients with suspected PHEO, statistical analysis of 24-hour urinary metanephrine and normetanephrine excretion test was performed, as well as a blood test for CrA, in groups that included patients without PHEO, with primary tumor or its recurrence, confirmed according to MSCT and/or scintigraphy with MIBG and/or the clonidine suppression test.

Results. The parameters of efficiency of these methods were calculated by groups and it was noted that the lowest sensitivity of the CrA determination method was observed in the group with recurrence of PHEO (43.8\%), their exclusion from the entire sample didn't change specificity of the method and it remained at a high level ( $85.45 \%$ ), though sensitivity significantly increased up to $87.1 \%$. Sensitivity of determining 24-hour urinary metanephrine excretion also increased significantly up to $96.8 \%$, with $98.2 \%$ of specificity. The correlation between diameter of the tumor and its secretory activity was identified: small - with CrA level (rho 0.491) and strong - with total level of methylated catecholamines (rho 0.765$)$. False positive results were more often observed in patients present with other neuroendocrine tumors $(37.5 \%)$, as well as those taking proton-pump inhibitors $(43.75 \%)$. The sensitivity and specificity of $\mathrm{CrA}$ determining method in the group of patients with methanephrins elevated within "gray zone" appeared to be 50 and $86.1 \%$, respectively.

Conclusion. A blood test for CrA can be recommended as a confirmatory test for diagnosing PHEO in cases of questionable methylated catecholamines indicators or in cases of suspected relapse of PHEO. The use of the test as a first-line method is only possible if there is no possibility to study methylated catecholamines. When interpreting CrA level, it is necessary to take into account the conditions that may cause false-positive results.
\end{abstract}

Keywords: pheochromocytoma, chromogranin A, methanephrine, normetanephrine, catecholamines

For citation: Yukina MYu, Karpova PL, Troshina EA, Platonova NM, Beltsevich DG. Chromogranin A in diagnosis of pheochromocytoma (comparative analysis). Terapevticheskii Arkhiv (Ter. Arkh.). 2021; 93 (4): 389-396. DOI: 10.26442/00403660.2021.04.200678

\section{Информация об авторах / Information about the authors}

Юкина Марина Юрьевна - к.м.н., вед. науч. сотр. отА. терапевтической эндокринологии. Тел.: +7(926)267-47-71;

e-mail: kuronova@yandex.ru; ORCID: 0000-0002-8771-8300

Карпова Покина Аьвовна - врач-ординатор.

ORCID: 0000-0002-2704-1027

Трошина Екатерина Анатольевна - ч^.-кор. РАН, А.м.н., профр., зав. отА. терапевтической эндокринологии, зам. Аир. по координации энАокринологической службы. ORCID: 0000-0002-8520-8702

Платонова Нахежьа Михайловна - $\Delta$. м.н., гл. науч. сотр. отА. терапевтической эндокринологии. ORCID: 0000-0001-6388-1544

Бельцевич $\Delta$ митрий Германович - А.м.н., гл. науч. сотр. отА. хирургии. ORCID: 0000-0001-7098-4584
Marina Yu. Yukina. E-mail: kuronova@yandex.ru; ORCID: 0000-0002-8771-8300

Polina L. Karpova. ORCID: 0000-0002-2704-1027

Ekaterina A. Troshina. ORCID: 0000-0002-8520-8702

Nadezhda M. Platonova. ORCID: 0000-0001-6388-1544

Dmitry G. Beltsevich. ORCID: 0000-0001-7098-4584 


\section{Введение}

Феохромоцитома (ФХЦ) и параганглиома (ПГ) - редкие гетерогенные опухоли из хромаффинных клеток, происходящие соответственно из мозгового слоя надпочечников или вненадпочечниковой хромаффинной ткани симпатических паравертебральных ганглиев грудной клетки, брюшной полости и таза. Опухоль надпочечниковой локализации обычно обнаруживается у $80-85 \%$ пациентов, а экстраадреналовые образования - у 10-20\% [1]. В целом катехоламин-секретирующие опухоли встречаются примерно у $0,3-0,5 \%$ пациентов с артериальной гипертензией (АГ) [2]. По оценкам, ежегодная заболеваемость составляет приблизительно 0,6 на 100 тыс. человек в год [3]. ФХЦ может возникать в любом возрасте, но наиболее часто - на 3-5-м десятилетии, с приблизительно одинаковым распределением между мужчинами и женщинами [4]. Около 10\% ФХЦ и $20 \%$ ПГ злокачественные и характеризуются плохим прогнозом выживаемости. Примерно в 25-35\% случаев ФХЦ может быть результатом мутаций и наследоваться как аутосомно-доминантный признак. С данным заболеванием ассоциировано более 14 генов. Некоторые наследственные случаи могут возникать в рамках таких комплексных состояний, как множественная эндокринная неоплазия (МЭН) 2А и 2В типа, синдром фон Гиппеля-Линдау, нейрофиброматоз или синдромы семейной ПГ 1, 3 или 4-го типа или же как семейная изолированная ФХЦ [5].

ФХЦ синтезирует катехоламины (адреналин, норадреналин, реже - дофамин), отдельно или в комбинации. Повышенная выработка данных веществ определяет спектр потенциальных симптомов, которые включают в себя классическую триаду: АГ, сердцебиение и обильное потоотделение. При этом характерны такие симптомы, как тревога и приступы паники, которые распространены и среди населения в целом, что затрудняет выявление заболевания. Для создания секреторных везикул с катехоламинами, а также их экзоцитоза необходимо наличие специальных нейроэндокринных секреторных белков - гранинов, к которым в том числе относится и хромогранин А (ХрA). В дополнение к основной функции хромогранинов - гранулогенезу ХрА является также предшественником многих функциональных пептидов, таких как вазостатин, панкреастатин, парастатин, серпенин и другие, которые выполняют различные физиологические нейроэндокринные функции. ХрА наиболее активно секретируется в хромаффинных клетках мозгового слоя надпочечников, но также присутствует и в других нейроэндокриноцитах, располагающихся в желудочно-кишечном тракте, поджелудочной железе, околощитовидных железах, органах репродуктивной системы, адипоцитах, сердечно-сосудистой и иммунной системе. ХрА, являясь ключевым гормоном нейроэндокринных клеток, часто секретируется нейроэндокринными опухолями (НЭО), в том числе и ФХЦ. В связи с этим измерение его концентрации в крови дает информацию для их выявления, оценки прогноза и мониторинга пациентов [6].

Согласно рекомендациям Международного общества эндокринологов, основой современной лабораторной диагностики ФХЦ является определение повышенных уровней свободных метанефринов (МН) в плазме или фракционированных МН в суточной моче (МН и норметанефрина - НMН) [7]. Данные вещества являются метаболитами (метилированными производными) катехоламинов. Определение немети- лированных гормонов не используется в связи с быстрым разрушением и выведением их из плазмы (менее 1 мин), что делает данный метод ненадежным и малоинформативным. Чувствительность и специфичность метода определения метилированных катехоламинов (МКА) в крови составляют 96 и $85 \%$ соответственно, в то время как анализ суточной мочи обладает большей специфичностью $(99,7 \%)$ при меньшей чувствительности $(87,5 \%)$. Оба метода являются эталонными и необходимы для достоверной верификации диагноза. Тем не менее их выполнение сопряжено с определенными трудностями, которые могут привести к неточному результату. Так, сбор суточной мочи некомфортен для пациентов, требует использования соляной кислоты в качестве консерванта, что может быть небезопасным. Кроме того, не исключена ошибка в самой методике сбора или подсчете объема мочи, а на результат могут влиять некоторые пищевые продукты и лекарственные препараты. Техника проведения анализа для определения МКА в плазме подразумевает нахождение пациента в горизонтальном положении в течение 30 мин непосредственно перед забором крови, что не всегда возможно в амбулаторных условиях, а сам анализ осуществляется лишь в небольшом количестве лабораторий в нашей стране. При получении недостаточных данных для постановки диагноза (повышение МКА в пределах «серой зоны», когда имеет место менее чем 3-кратное повышение показателей относительно верхнего референсного порога) пациентов либо оставляют под динамическим наблюдением и повторно проводят анализ через 3-6 мес, что не исключает развития катехоламинового криза за этот период, либо применяют пробу с клонидином на основе исследования МКА в анализе крови до и через 3 ч после приема препарата, с соответствующими техническими сложностями и дополнительными рисками. Все эти недостатки делают крайне актуальным поиск других методов диагностики ФХЦ, наиболее удобных и простых в исполнении как для врачей, так и для пациентов, а также обладающих высокой точностью при минимальных затратах. Анализ крови на ХрА рассматривается как один из способов диагностики, соответствующий данным критериям.

Определение уровня ХрА в плазме в настоящее время наиболее часто используется для диагностики гастроинтестинальных НЭО, в особенности для мониторинга их рецидива после радикального лечения. Данный метод впервые для диагностики ФХЦ предложили R. Hsiao и соавт., по результатам проведенного исследования показатели эффективности теста оказались относительно высокими: чувствительность составила $83 \%$, специфичность 96\%. Кроме того, выявлена положительная корреляция ХрА с массой опухоли, ее экскреторной активностью [8]. В дальнейшем эти данные подтверждены во многих исследованиях. Так, в работах R. Bílek и соавт. показана значимая связь между концентрацией ХрА и наличием ФХЦ. В их работе 2008 г. [9] проводился сравнительный анализ лабораторных данных в группах пациентов с ФХЦ непосредственно перед оперативным лечением $(n=25$, в том числе 3 пациента с синдромом МЭН 2-го типа ) и через 1 год после ( $n=13)$, с медуллярным раком щитовидной железы, но без ФХЦ ( $n=22)$, с врожденной дисфункцией коры надпочечников $(n=33)$, контрольная же группа включала пациентов с наличием гормонально-неактивных опухолей надпочечников $(n=11), 4$ пациентов с АГ и 9 - с различными заболеваниями щитовидной железы. Пациенты с ФХЦ имели наибольшие значения уровня ХрА по сравнению со всеми остальными. Также незначительное повышение данного показателя 
отмечено в группе с медуллярным раком, по сути являющимся НЭО. Авторы в дальнейшем продолжили свои исследования по диагностике ФХЦ, показатели эффективности метода определения ХрА для выявления данного заболевания составили 90-93 и 92-96\% для чувствительности и специфичности соответственно, при этом значения по определению МКА ненамного выше [10, 11].

Однако некоторые исследователи не подтверждают столь высокую диагностическую ценность метода. Например, в работе N. Unger и соавт. ХрА показал относительно низкие чувствительность и специфичность $(73,9$ и 74,2\%) при пороговом уровне 215 мкг/л. Кроме того, авторы определили высокий уровень ХрА у 40\% пациентов после оперативного лечения [12]. Такие результаты могут быть связаны, по мнению авторов, с наличием у этих пациентов сниженной почечной функции или приемом ингибиторов протонной помпы (ИПП), что не учитывалось при проведении анализа данных. Кроме того, не учитывали и возможность наличия других НЭО. В работе же Е. Al-Risi и соавт. ХрА изучался как маркер различных НЭО, при этом анализировали и сопутствующие состояния пациентов, которые могут влиять на его уровень. Показано, что чувствительность составила $84,2 \%$, а специфичность - 78,2\%, у пациентов с ложноположительным результатом диагностировали гипертоническую болезнь, хроническую болезнь почек, сердечную недостаточность, язвенную болезнь, хроническую диарею, гипотиреоз, астму и сахарный диабет, некоторые пациенты применяли ИПП [13]. Часть работ показывает, что ХрА оценивает лишь факт наличия секреторной активности НЭО, но не позволяет определить характер образования. По данным метаанализа, включающего 13 исследований (1260 пациентов с НЭО и 967 здоровых добровольцев), чувствительность метода определили в 73\%, 95\% доверительный интервал (71\%; 76\%), а специфичность 95\% (93\%; 96\%) [14] с относительно низкой прогностической ценностью [15]. Определено, что чувствительность метода для прогнозирования локализации НЭО крайне низкая (43\%), немного выше для выявления метастазов - 57\% [16].

Относительно высокая чувствительность метода определения ХрА при низкой специфичности позволяет некоторым авторам предлагать его в качестве первичной диагностики (исключения) ФХЦ, с последующим формированием более малочисленной группы пациентов для наблюдения и выявления заболевания, что снизило бы нагрузку на амбулаторное звено при минимальном риске пропуска потенциальных больных [17]. Например, в исследовании P. Glinicki и соавт. показано, что у всех пациентов с ФХЦ наблюдался повышенный уровень ХрА, при этом встречались случаи его повышения при других состояниях (образования, продуцирующие кортизол) [18]. Кроме того, метод первичной диагностики может помочь избежать проведения необоснованных визуализирующих исследований и оперативных вмешательств пациентам, которые в них не нуждаются, что и показали в своей работе L. Giovanella и соавт. Анализ лабораторных данных 348 пациентов выявил повышенный ХрА у 39 из них, при этом у 18 в дальнейшем диагностирована ФХЦ по результатам сцинтиграфии с метайодбензилгуанидином. Все 18 характеризовались значительным повышением уровня данного маркера, при этом пациенты со значениями в пределах референсного интервала действительно не нуждались в дальнейшем обследовании, так как заболевания не имели [19].
Таким образом, анализ литературных данных мировых исследований относительно метода определения ХрА в плазме для выявления ФХЦ показал неоднозначные данные о его эффективности и надежности. Как и на все современные эталонные диагностические маркеры данного заболевания, на результат ХрА также может влиять наличие определенных сопутствующих состояний, биохимически и морфологически сходных с ФХЦ. Но при этом методика очевидно лишена изложенных недостатков рутинного скрининга, так как проведение теста не требует много времени, специальной подготовки, неопасно и экономически выгодно.

С учетом актуальности проблемы поставлены следующие задачи: проанализировать первичные данные уровня ХрА у пациентов с ФХЦ и гормонально-неактивными образованиями надпочечников; установить чувствительность и специфичность метода определения ХрА в диагностике ФХЦ в сравнении с определением МКА в суточной моче; выявить группы пациентов, для которых применение данного метода наиболее/наименее целесообразно.

\section{Материалы и методы}

В сравнительное аналитическое исследование включены 157 больных с подозрением на ФХЦ. Наиболее частыми причинами поиска ФХЦ являлись: АГ, образования надпочечников, панические/тревожные расстройства и обследование пациентов с медуллярным раком щитовидной железы перед операцией. Всем пациентам проведено исследование МКА в анализе суточной мочи (эталонный метод) и ХрА в анализе крови. Диагноз считался лабораторно подтвержденным при показателе МН или/и НМН выше 3-кратного уровня верхнего референса (936 и 1335 мкг/сут соответственно). Значения МН и НМН в пределах 3-кратного повышения расценены как «серая зона» (для анализа использовался наибольший показатель - либо МН, либо НМН). Положительным результатом анализа на ХрА считался его уровень выше 2 нмоль/л.

Все пациенты (1-я группа) разделены на 3 основные группы с учетом наличия первично диагностированной ФХЦ (2-я группа), рецидива ФХЦ (3-я группа) и отсутствия данного заболевания (4-я группа).

На основании полученных данных рассчитаны показатели эффективности методов (чувствительность, специфичность, прогностическая ценность положительного и отрицательного результатов) и проведен их сравнительный анализ как в целой выборке, так и отдельно по группам.

Подтверждение наличия ФХЦ основывалось на данных лабораторно-инструментального обследования (мультиспиральная компьютерная томография органов брюшной полости, и/или сцинтиграфия с метайодбензилгуанидином, и/или проба с клонидином). Большинству пациентов с ФХЦ проведено оперативное лечение с последующим гистологическим исследованием послеоперационного материала.

Статистическая обработка результатов осуществлялась с использованием программ Microsoft Excel XLSTAT, Statistica 10.0 (StatSoft Inc., США), применялся общепринятый метод статистического анализа - ROC-анализ, о статистической значимости различий качественных признаков судили по критерию Мак-Немара, а для оценки связи между параметрами применялся корреляционный анализ по Спирмену. Результаты представлены в виде медианы $(\mathrm{Me})$ с интерквартильным размахом [25-й; 75-й перцентили]. Различия считались статистически значимыми при $p<0,05$. 
Таблица 1. Характеристики эффективности методов Аиагностики ФХШ в 1-й группе $(n=157)$

\begin{tabular}{lccc}
\hline Параметр & $\mathbf{X р А}, \boldsymbol{\%}$ & МКА, $\%$ & $\boldsymbol{p}$ \\
\hline \multirow{2}{*}{$\mathrm{Se}$} & 72,3 & 70,2 & 0,68 \\
& {$[58,8 ; 83,4]$} & {$[57,4 ; 82,2]$} & \\
$\mathrm{Sp}$ & 85,5 & 98,2 & 0,0001 \\
& {$[77,6 ; 91,2]$} & {$[93 ; 99,8]^{*}$} & \\
+PV & 87,9 & 88,5 & 0,87 \\
-PV & 68 & $94,3 *$ & $<0,05$ \\
\hline
\end{tabular}

*Здесь и далее - статистически значимое различие.

Таблица 2. Характеристики эффективности методов Аиагностики ФХШ во 2-й группе $(n=31)$

\begin{tabular}{lccc}
\hline Параметр & ХрА, $\%$ & МКА, $\%$ & $\boldsymbol{p}$ \\
\hline $\mathrm{Se}$ & 87,1 & 96,8 & 0,17 \\
$\mathrm{Sp}$ & - & - & - \\
$+\mathrm{PV}$ & 100 & 100 & 1 \\
$\mathrm{-PV}$ & 0 & 0 & 1 \\
\hline
\end{tabular}

Таблица 3. Характеристики эффективности методов Аиагностики ФХШ в 3-й группе (n=16)

\begin{tabular}{lccc}
\hline Параметр & ХрА, $\%$ & МКА, $\%$ & $\boldsymbol{p}$ \\
\hline $\mathrm{Se}$ & 43,8 & 18,8 & 0,14 \\
$\mathrm{Sp}$ & - & - & - \\
$+\mathrm{PV}$ & 100 & 100 & 1 \\
-PV & 0 & 0 & 1 \\
\hline
\end{tabular}

Таблица 4. Характеристики эффективности методов Аиагностики ФХШ в 4-й группе $(n=110)$

\begin{tabular}{lccc}
\hline Параметр & ХрА, $\%$ & МКА, $\%$ & $\boldsymbol{p}$ \\
\hline $\mathrm{Se}$ & - & - & - \\
$\mathrm{Sp}$ & 85,5 & $98,2^{*}$ & 0,0007 \\
$+\mathrm{PV}$ & 0 & 0 & 1 \\
-PV & 100 & 100 & 1 \\
\hline
\end{tabular}

Таблица 5. Характеристики эффективности метода определения ХрА (1, 5-я группы)

\begin{tabular}{lccc}
\hline Параметр & $\begin{array}{c}\mathbf{1 - я} \text { группа, } \\
\text { \% }\end{array}$ & $\begin{array}{c}\text { 5-я группа, } \\
\mathbf{\%}\end{array}$ & $\boldsymbol{p}$ \\
\hline \multirow{2}{*}{$\mathrm{Se}$} & 72,3 & 87,1 & 0,018 \\
& {$[58,8 ; 83,4]$} & {$[71,2 ; 95,5]^{*}$} & \\
$\mathrm{Sp}$ & 85,5 & 85,5 & 1 \\
$\mathrm{HV}$ & {$[77,6 ; 91,2]$} & {$[77,6 ; 91,2]$} & 1 \\
-PV & 87,9 & $62,8^{*}$ & $<0,05$ \\
\hline
\end{tabular}

\section{Результаты}

Исследование ХрА в анализе крови и МКА в анализе суточной мочи проведено 157 пациентам с подозрением на ФХЦ: 111 женщинам и 46 мужчинам, медиана возраста 47,5 года [15; 78]. У 31 пациента впоследствии диагностирована первичная хромаффинная опухоль, у 16 - выявлен рецидив ФХЦ, у 110 - диагноз не подтвержден. Таким

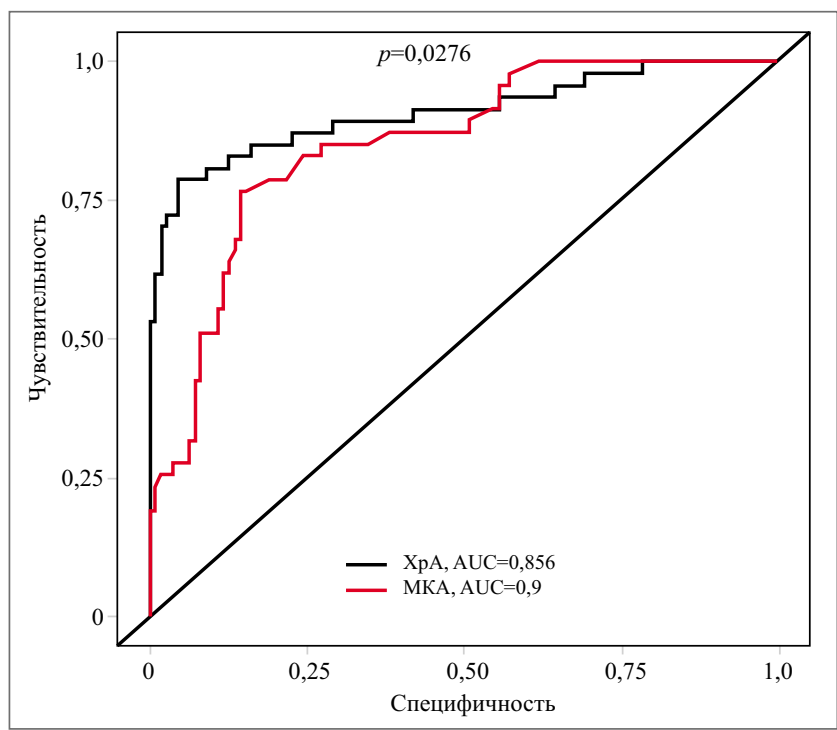

Рис. 1. Эфффективность методов определения ХрА в анамизе крови и МКА в анализе суточной мочи в 1-й группе.

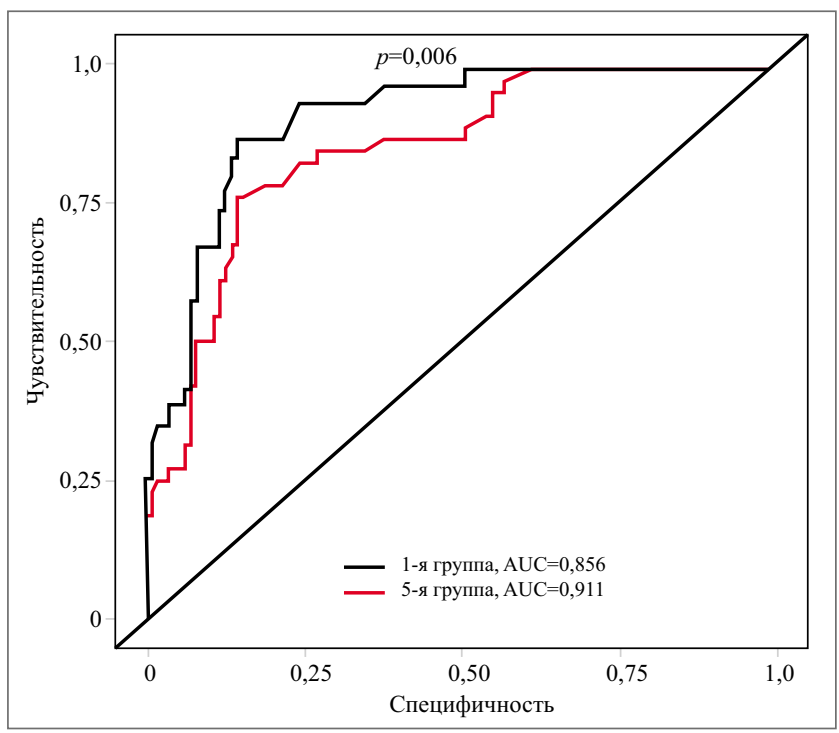

Рис. 2. Эфффективность метода определения ХрА по 1 и 5-й группам.

образом, среди пациентов с подозрением на ФХЦ опухоль подтверждена в $42,7 \%$ случаев.

Рассчитаны показатели эффективности методов по основным группам (табл. 1-4, рис. 1).

При проведении анализа отмечено, что наиболее низкие цифры чувствительности для метода определения ХрА наблюдаются в 3-й группе. При исключении из 1-й группы 3-й группы (сформирована 5-я группа; $n=141$ ) получены следующие данные: специфичность метода определения ХрА сохранилась на высоком уровне и составила $85,45 \%$, при этом чувствительность значимо возросла до $87,1 \%$ (табл. 5, рис. 2).

В 5-й группе также значимо повысился показатель чувствительности метода определения МКА в суточной моче до $96,8 \%$ (табл. 6, рис. 3 ).

Таким образом, отмечено, что в 5-й группе показатели эффективности метода определения ХрА приблизились к 
Таблица 6. Характеристики эффективности метода определения МКА по 1 и 5-й группам

\begin{tabular}{lccc}
\hline Параметр & $\begin{array}{c}\text { 1-я группа, } \\
\text { \% }\end{array}$ & $\begin{array}{c}\text { 5-я группа, } \\
\text { \% }\end{array}$ & $\boldsymbol{p}$ \\
\hline \multirow{2}{*}{$\mathrm{Se}$} & 70,2 & 96,8 & $<0,05$ \\
& {$[57,4 ; 82,2]$} & {$[83 ; 100]^{*}$} & \\
$\mathrm{Sp}$ & 98,2 & 98,2 & 1 \\
$\mathrm{HVV}$ & {$[93 ; 99,8]$} & {$[93 ; 99,8]$} & \\
-PV & 88,5 & 93,8 & 0,11 \\
\hline
\end{tabular}

Таблица 7. Сравнение характеристик эффективности методов в 5-й группе

\begin{tabular}{lccc}
\hline Параметр & ХрА, \% & МКА, $\%$ & $\boldsymbol{p}$ \\
\hline \multirow{2}{*}{ Se } & 87,1 & 96,8 & 0 \\
& {$[71,2 ; 95,5]^{*}$} & {$[83 ; 100]^{*}$} & 0,003 \\
Sp & 85,5 & 98,2 & \\
& {$[77,6 ; 91,2]$} & {$[93 ; 99,8]^{*}$} & 0,0001 \\
+PV & 62,8 & $93,8^{*}$ & $<0,05$ \\
-PV & 95,9 & $100^{*}$ & 0,016 \\
\hline
\end{tabular}

методу определения МКА в моче, хотя и остались на более низком уровне (табл. 7, рис. 4).

С целью выяснения причины ложноотрицательных результатов определения ХрА у пациентов с ФХЦ (2 и 3-я группы; $n=47)$ больные повторно разделены на группы: с ложноотрицательным (6-я группа; $n=13)$ и с истинноположительным результатом (7-я группа; $n=34)$. Далее в этих группах проведен сравнительный анализ относительно диаметра опухоли (или сумма диаметров опухолей, если более одного узла; при наличии трех или двух размеров принимался к расчету бо́льший), уровня и типа экскреции МН и НМН (уровень - суммарно; тип экскреции определялся гормоном, экскреция которого больше другого в 1,5 раза, либо как «смешанный», если меньше). Согласно полученным данным, в 6-й группе диаметр опухоли и уровень МКА значимо меньше, чем в 7-й группе: (25 мм [14; 36]) против 51 мм $[35,75 ; 66,75]$ при $p=0,0005$ для размера образований и 3184,9 мкг/сут [1457,4; 6453,8] против 823,7 мкг/сут [382,7; $1325,1]$ при $p=0,001$ для МКА; определена слабая корреляция между уровнем ХрА и диаметром опухоли (rho $=0,491$, $p<0,05)$, а также сильная с уровнем МКА (rho $=0,765, p<0,05)$. Данные взаимосвязи наглядно представлены на рис. 5, 6.

Кроме того, между 6 и 7-й группами не выявлено значимых различий по преимущественному типу экскреции, наиболее часто отмечался норметанефриновый в 7-й группе (табл. 8).

С целью выяснения причины ложноположительных результатов определения ХрА у пациентов без ФХЦ (4-я группа; $n=110$ ) больные разделены на группы: с ложноположительным (8-я группа; $n=16)$ и с истинноотрицательным результатом (9-я группа; $n=94)$. Далее в этих группах

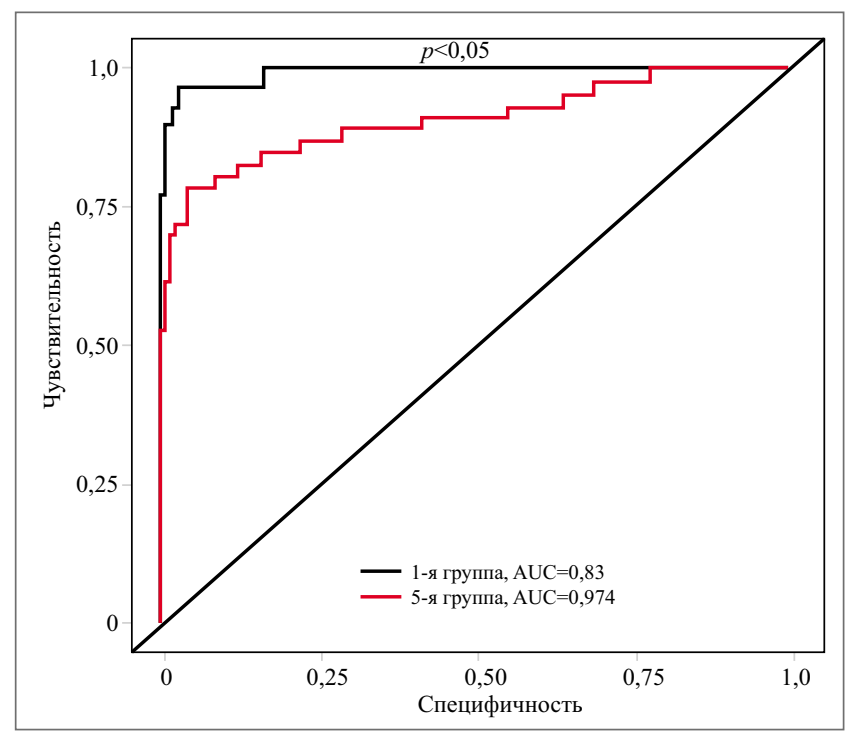

Рис. 3. Эфффективность метода определения МКА в 1 и 5-й группах.

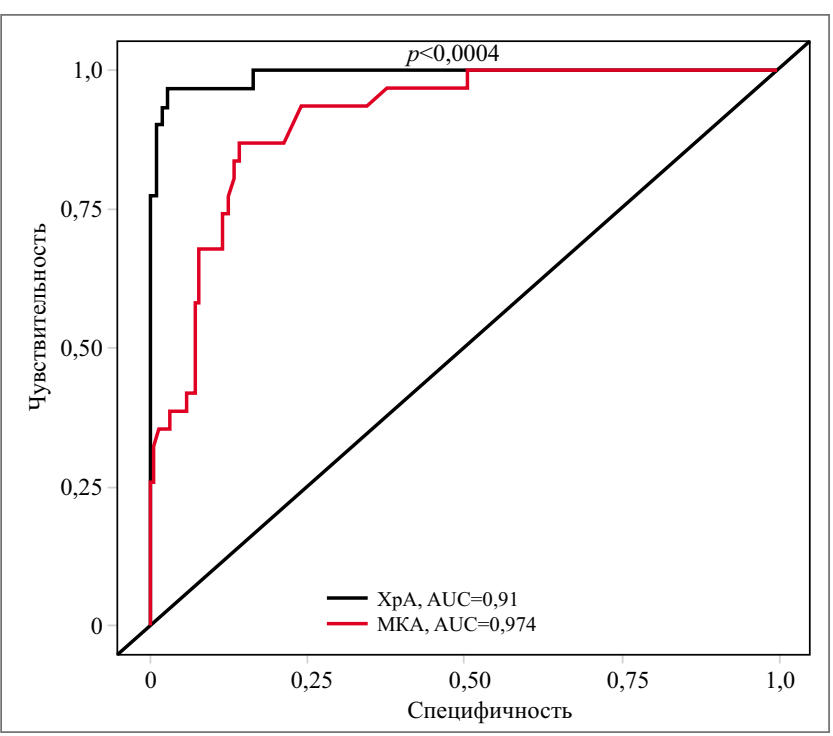

Рис. 4. Эфффективность методов определения ХрА в анализе крови и МКА в анализе суточной мочи в 5-й группе.

проведен сравнительный анализ относительно уровня экскреции МН и НМН (суммарно МН и НМН), показателей МКА в пределах «серой зоны», приема ИПП, наличия других НЭО. Согласно полученным данным, в 8-й группе другие НЭО встречались в 37,5\% случаев, 43,75\% пациентов принимали ИПП. Однако уровень МКА в этой группе значимо не отличался от 9-й группы $(p=0,9)$. Всего 31 (28,2\%) пациент из 9-й группы имел показатели МКА в пределах «серой зоны», в 8-й группе статистически значимо чаще встречались такие результаты: у $5(31,3 \%)$ человек, $p<0,05$. Наиболее часто

Таблица 8. Преимушественный тип экскреции в 6 и 7-й группах

\begin{tabular}{lccc}
\hline Тип экскреции & 6-я группа & 7-я группа & $p$ (между группами) \\
\hline Норметанефриновый & $69,20 \%$ & $67,6 \% *$ & 0,9 \\
Метанефриновый & $0 \%$ & $14,7 \%$ & 0,15 \\
Смешанный & $30,70 \%$ & $17,7 \%$ & 0,34 \\
$p$ & 0,06 & 0,001 & \\
\hline
\end{tabular}




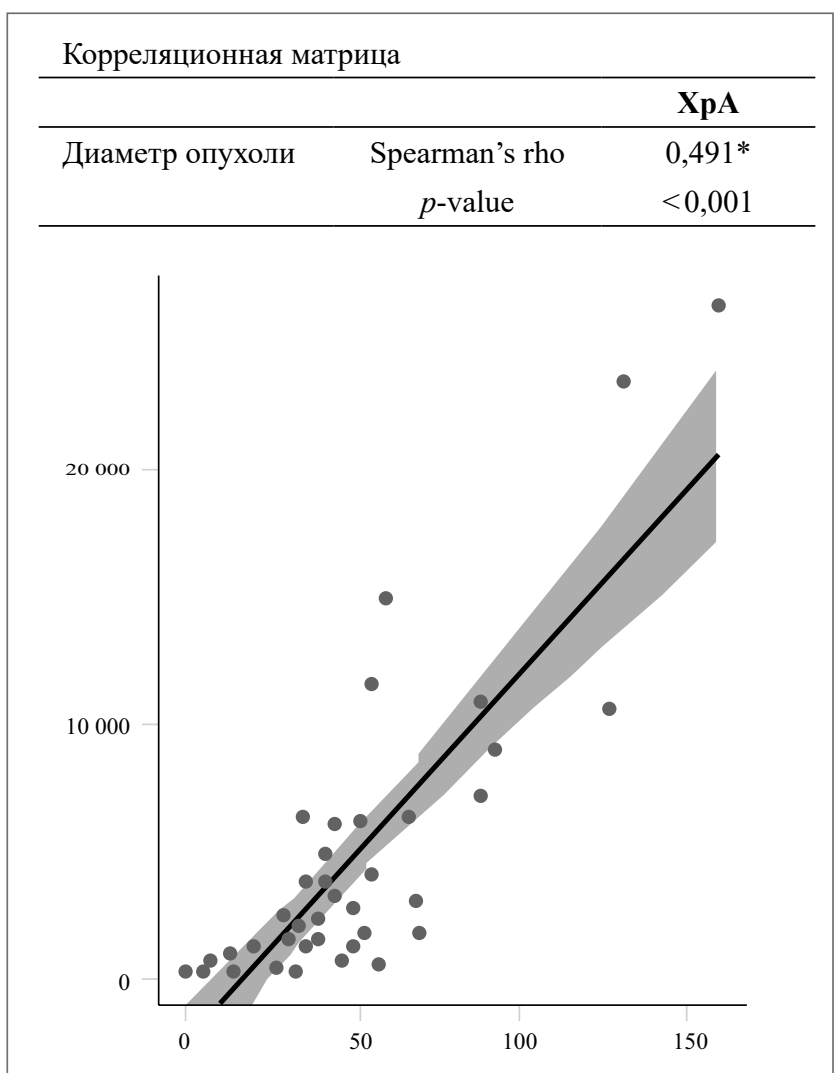

Рис. 5. Корреляция между размером опухоли и уровнем ХрА в во 2 и 3-й группах.

\begin{tabular}{|c|c|c|}
\hline \multicolumn{3}{|c|}{ Корреляционная матрица } \\
\hline & & $\mathbf{X p A}$ \\
\hline \multirow[t]{2}{*}{ MKA } & Spearman's rho & $0,765^{*}$ \\
\hline & $p$-value & $<0,001$ \\
\hline
\end{tabular}

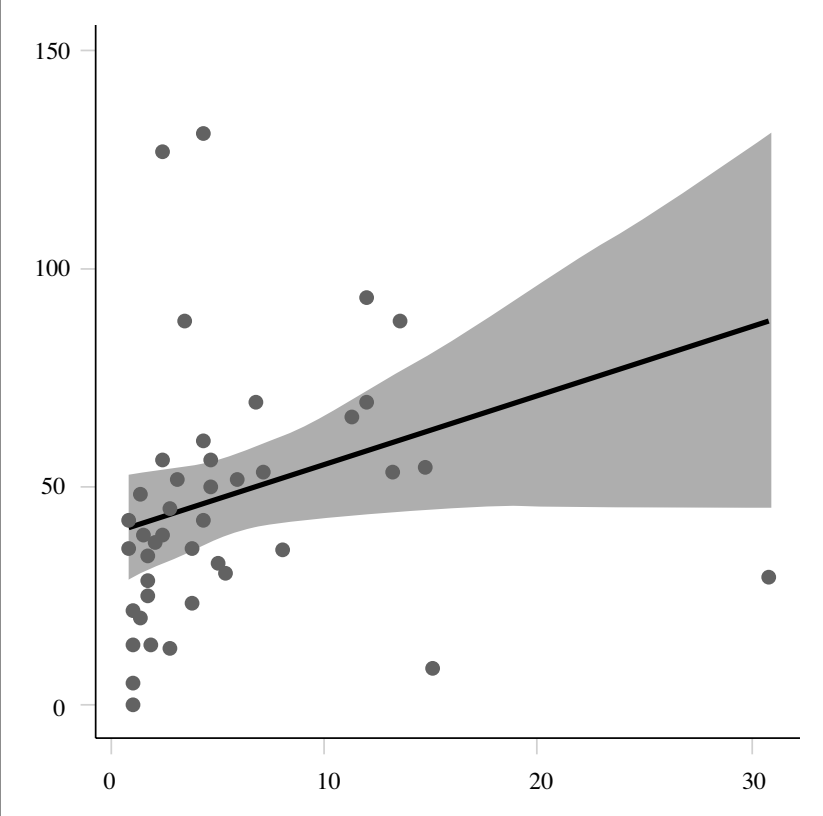

Рис. 6. Корреляция межАу уровнем ХрА и суммарным уровнем МКА во 2 и 3-й группах.
Таблица 9. Преимушественный тип экскреции в 9-й группе

\begin{tabular}{lc}
\hline Тип экскреции & 9-я группа \\
\hline Норметанефриновый & $72,2 \% *$ \\
Метанефриновый & $2,8 \%$ \\
Смешанный & $25 \%$ \\
$p$ & 0,002 \\
\hline
\end{tabular}

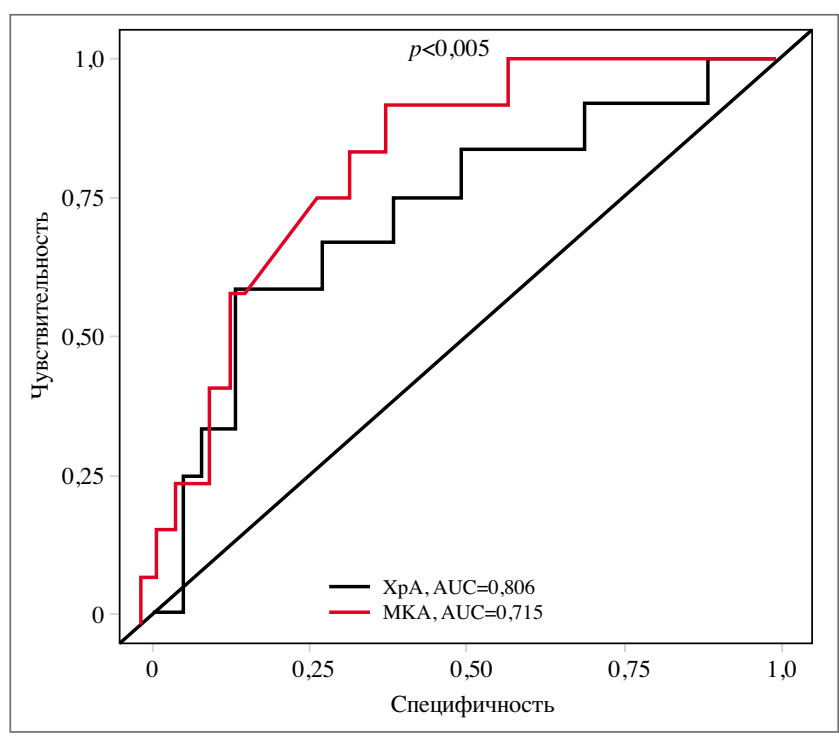

Рис. 7. Эффективность методов определения ХрА в анализе крови и МКА в анализе суточной мочи в 10-й группе.

среди пациентов со значениями МКА в «серой зоне» отмечался норметанефриновый тип экскреции (табл. 9).

Кроме того, уровень ХрА среди пациентов 4-й группы оказался достоверно более высоким у пациентов с полученными результатам МКА в пределах «серой зоны» $(p=0,000336)$ и составил 2,25 [0,3; 9], в то время как у пациентов с нормальными показателями МКА он составил 0,87 [0,1; 1,7].

Для проверки эффективности метода определения ХрА в качестве уточняющего теста при получении результата анализа суточной мочи на МКА в пределах «серой зоны» в исследовании выделена группа таких пациентов (10-я группа, $n=48)$. Чувствительность и специфичность метода в 10 -й группе оказалась 50 и 86,1\% соответственно (рис. 7).

\section{Обсужнение}

Исследование ХрА в анализе крови и МКА в анализе суточной мочи проведено 157 пациентам с подозрением на ФХЦ: 111 женщинам и 46 мужчинам, средний возраст в группе составил 47,5 года [20]. Средний возраст выявления ФХЦ соответствует общемировым данным, которые обозначают 3-5-е десятилетие жизни [4]. Преимущество для лиц женского пола также отмечается многими авторами, но в незначительной степени. По нашему мнению, смещение выборки в данном случае связано всего лишь с традиционно более внимательным отношением к своему здоровью женщин, в отличие от мужчин.

В общей группе пациентов с подозрением на ФХЦ опухоль подтверждена в 42,7\% случаев. Здесь важно акцентировать внимание на смещение выборки пациентов в связи 
с тем, что обращаемость в специализированный стационар уже сама по себе носит ограничительный характер: бо́льшая часть пациентов с АГ и инциденталомой уже первично осмотрена специалистами, и зачастую не одним (терапевт, кардиолог, рентгенолог, эндокринолог, онколог). В действительности распространенность хромаффинной опухоли гораздо меньше: 1 пациент на 2,5-6,5 тыс. населения [1].

На основании полученных данных рассчитаны показатели эффективности методов по группам и отмечено, что наиболее низкие цифры чувствительности наблюдались в группе с рецидивом ФХЦ - 43,8\% для ХрА и 18,8\% - для МКА. При исключении этой группы из всей выборки чувствительность метода определения ХрА значимо возросла с 72,3 до 87,1\%, но возросла и для определения МКА с 70 до 96,8\%. При этом специфичность метода определения ХрА и в общей выборке, и в группе пациентов с показателями МКА в пределах «серой зоны» оставалась относительно высокой - 86\% (98,2\% для МКА). Таким образом, несмотря на перечисленные недостатки метода определения МКА в анализе суточной мочи, он остается методом 1-го ряда в диагностике ФХЦ. Учитывая относительно высокую специфичность, метод определения ХрА может применяться как подтверждающий тест при подозрении на рецидив ФХЦ или при сомнительных показателях МКА, но как единственный и первичный - только при отсутствии возможности исследования МКА.

Согласно полученным данным в группе с ложноотрицательным результатом диаметр опухоли и уровень МКА значимо меньше, чем в группе с истинноположительным, определена средней силы корреляция между уровнем ХрА и диаметром опухоли, а также сильная - с уровнем экскреции МКА (положительная корреляция уровня МКА и ХрА получена и в других группах). Таким образом, выявление крупной опухоли надпочечника при незначительном повышении ХрА сомнительно относительно диагноза ФХЦ. Обычно крупные опухоли отличаются значимо повышенной секрецией и, соответственно, яркими клиническими проявлениями, а такие пациенты как раз и попадают под прицельное обследование. Иначе дело обстоит с пациентами, у которых, например, выявлен медуллярный рак щитовидной железы, отсутствуют клинические признаки, подозрительные на ФХЦ, и в рамках предоперационного обследования им необходимо исключить ФХЦ. Таким пациентам следует применять более чувствительный метод.

Похожие данные относительно корреляции между диаметром опухоли и ее секрецией получены в исследовании на группе пациентов с ФХЦ при определении МКА в суточной моче и обусловлены едиными гистогенетическими особен- ностями нейроэндокринной ткани, продуцирующей как катехоламины, так и ХрА [21]. Положительная связь между размером опухоли и уровнем гормональной секреции часто описывается для высокодифференцированных опухолей с большим количеством секреторных гранул [22]. В связи с этим можно предположить, что при злокачественной ФХЦ могут отмечаться нормальные или сниженные показатели ХрА. Для подтверждения данной гипотезы требуется проведение дополнительного исследования, так как в нашей выборке злокачественная ФХЦ отсутствовала (точнее, не выявлено метастатическое поражение).

Преимущественное большинство пациентов в нашей работе с норметанефриновым типом экскреции и значимо по типу экскреции не различались по группам. Метанефриновый тип встречается редко и характерен для ФХЦ при МЭН 2-го типа [23]. Наиболее часто среди пациентов со значениями МКА в «серой зоне» также отмечался норметанефриновый тип экскреции. Известно, что повышение в пределах «серой зоны» МН в большинстве случаев обозначает именно опухолевую секрецию, а НМН, напротив, физиологическую активацию симпатической нервной системы [24].

В нашей работе большинство случаев ложноположительного результата ожидаемо получено у пациентов с другими НЭО и принимающих ИПП, что также согласуется с данными других авторов [25].

\section{ЗакАючение}

Таким образом, определение ХрА в анализе крови является относительно точным, быстрым, безопасным, легким в исполнении и недорогим дополнительным методом лабораторной диагностики ФХЦ. Анализ крови на ХрА можно рекомендовать в качестве подтверждающего теста для диагностики ФХЦ при сомнительных показателях МКА или при подозрении на рецидив ФХЦ. Применение теста в качестве метода 1-го ряда - только при отсутствии возможности исследования МКА.

При интерпретации результата анализа на ХрА необходимо учитывать состояния, вызывающие ложноположительное повышение, особенно при таких заболеваниях, как хроническая сердечная и почечная недостаточность, а также применение некоторых лекарственных средств (ИПП). Кроме того, важно помнить, что ХрА может быть маркером любой другой НЭО.

Авторы заявляют об отсутствии конфликта интересов.

\section{Список сокрашений}

АГ - артериальная гипертензия

ИПП - ингибиторы протонной помпы

МКА - метилированные катехоламины

МН - метанефрин

МЭН - множественная эндокринная неоплазия
НМН - норметанефрин

НЭО - нейроэндокринная опухоль

ПГ - параганглиома

ФХЦ - феохромоцитома

$\mathrm{XpA}$ - хромогранин А

\section{NИTEPATYPA/REFERENCES}

1. Chen H, Sippel RS, O'Dorisio MS, et al. The north american neuroendocrine tumor society consensus guideline for the diagnosis and management of neuroendocrine tumors: Pheochromocytoma, paraganglioma, and medullary thyroid cancer. Pancreas. 2010;39(6):77583. doi: 10.1097/MPA.0b013e3181ebb4f0

2. Omura M, Saito J, Yamaguchi K, et al. Prospective Study on the Prevalence of Secondary Hypertension among Hypertensive Patients
Visiting a General Outpatient Clinic in Japan. Hypertens Res Vol. 2003;27(3):193-202. doi: 10.1291/hypres.27.193

3. Neumann HPH, Young WFJr, Eng C. Pheochromocytoma and Paraganglioma. N Engl J Med. 2019;381(6):552-65. doi: 10.1056/NEJMra1806651

4. Mannelli M, Lenders JWM, Pacak K, et al. Subclinical phaeochromocytoma. Best Pract Res Clin Endocrinol Metab. 2012;26(4):507-15. doi: 10.1016/j.beem.2011.10.008 
5. Gimm O, Koch CA, Januszewicz A, et al. The genetic basis of pheochromocytoma. Front Horm Res. 2004;31:45-60. doi: 10.1159/000074657

6. Zuber S, Wesley R, Prodanov T, et al. Clinical utility of chromogranin A in SDHx- related paragangliomas. Eur J Clin Invest. 2014;44(4):365-71. doi: $10.1111 /$ eci.12245

7. Lenders JWM, Duh QY, Eisenhofer G, et al. Pheochromocytoma and paraganglioma: An endocrine society clinical practice guideline. J Clin Endocrinol Metab. 2014;99(6):1915-42. doi: 10.1210/jc.2014-1498

8. Hsiao RJ, Parmer RJ, Takiyyuddin MA, O'Connor DT. Chromogranin A storage and secretion: sensitivity and specificity for the diagnosis of pheochromocytoma. Medicine (Baltimore). 1991;70(1):33-45. doi: 10.1097/00005792-199101000-00003

9. Bílek R, Ík LŠ̌̌, Ciprová V, et al. Chromogranin A, a member of neuroendocrine secretory proteins as a selective marker for laboratory diagnosis of pheochromocytoma. Physiol Res. 2008;57:171-9.

10. Bílek R, Zelinka T, Vlček P, et al. Radioimmunoassay of Chromogranin A and Free Metanephrines in Diagnosis of Pheochromocytoma. Physiol Res. 2017;66:397-408. doi: 10.33549/physiolres.933719

11. Bílek R, Zelinka T, Vlček P, et al. Deconjugated Urinary Metanephrine, Normetanephrine and 3-Methoxytyramine in Laboratory Diagnosis of Pheochromocytoma and Paraganglioma. Physiol Res. 2015;64:313-22. doi: 10.33549/physiolres.933109. PMID: 26680494

12. Unger N, Hinrichs J, Deutschbein T, et al. Plasma and Urinary Metanephrines Determined by an Enzyme Immunoassay, but not Serum Chromogranin A for the Diagnosis of Pheochromocytoma in Patients with Adrenal Mass. Exp Clin Endocrinol Diabetes. 2012;120(8):494500. doi: $10.1055 / \mathrm{s}-0032-1309007$

13. Al-risi ES, Al-essry FS. Chromogranin A as a Biochemical Marker for Neuroendocrine Tumors: A Single Center Experience at Royal Hospital, Oman. Oman Med J. 2017;32(5):365-70. doi: 10.5001/omj.2017.71

14. Yang X, Yang Y, Li Z, et al. Diagnostic value of circulating chromogranin a for neuroendocrine tumors: A systematic review and meta-analysis. PLoS One. 2015;10(4):1-14. doi: 10.1371/journal.pone.0124884

15. Kidd M, Bodei L, Modlin IM. Chromogranin A: any relevance in neuroendocrine tumors? Curr Opin Endocrinol Diabetes Obes. 2016. 2016;23(1):28-37. doi: 10.1097/MED.0000000000000215

16. Cimitan M, Buonadonna A, Cannizzaro R, et al. Somatostatin receptor scintigraphy versus chromogranin A assay in the management of patients with neuroendocrine tumors of different types: Clinical role. Ann Oncol. 2003;14(7):1135-41. doi: 10.1093/annonc/mdg279

17. Zawadzka-Leska SK, Radziszewski M, Malec K, Stadnik AUA. Predictive value of chromogranin a in a diagnosis towards Pheochromocytoma in adrenal incidentaloma. Endocr Care. 2016;12(4):437-42. doi: $10.4183 /$ aeb.2016.437

18. Glinicki P, Jeske W, Bednarek-Papierska L, et al. Chromogranin A (CgA) in adrenal tumours. Endokrynol Pol. 2013;64(5):358-62. doi: 10.5603/ep.2013.0018

19. Giovanella L, Ceriani L, Balerna M, et al. Diagnostic value of serum chromogranin-A combined with MIBG scintigraphy in patients with adrenal incidentalomas. Q J Nucl Med Mol Imaging. 2008;52(1):84-8.

20. De Jong WHA, Eisenhofer G, Post WJ, et al. Dietary influences on plasma and urinary metanephrines: Implications for diagnosis of catecholamineproducing tumors. J Clin Endocrinol Metab. 2009;94(8):2841-9. doi: $10.1210 /$ jc.2009-0303

21. Юкина М.Ю., Трошина Е.А.. Бельцевич Д.Г., и др. Феохромоцитома/параганглиома: клинико-генетические аспекты. Проблемы эндокринологии. 2013;3:19-26 [Yukina MY, Troshina EA, Beltsevich DG, et al. Pheochromocytoma/paraganglioma: clinical and genetic aspects. Problemy endokrinologii. 2013;3:19-2 (In Russ.)]. doi: 10.14341/probl201359319-26

22. Ardill JES, O'Dorisio TM. Circulating biomarkers in neuroendocrine tumors of the enteropancreatic tract: application to diagnosis, monitoring disease, and as prognostic indicators. Endocrinol Metab Clin North Am. 2010;39(4):777-90. doi: 10.1016/j.ecl.2010.09.001

23. Martucci VL, Pacak K. Pheochromocytoma and Paraganglioma: Diagnosis, Genetics, Management, and Treatment. Curr Probl Cancer. 2014;38(1):7-41. doi: 10.1038/jid.2014.371

24. Herman DS, Lam L, Taylor M, et al. Catecholamine metabolomic and secretory phenotypes in phaeochromocytoma. Endocr Relat Cancer. 2011;18(1):97-111. doi: 10.1038/jid.2014.371

25. Pregun I, Herszényi L, Juhász M, et al. Effect of proton-pump inhibitor therapy on serum chromogranin A level. Digestion. 2011;84(1):22-8. doi: $10.1159 / 000321535$

Статья поступила в редакцию / The article received: 20.04.2020

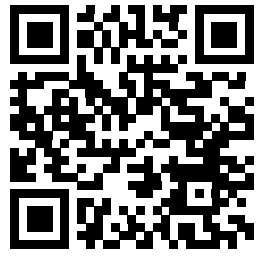

OMNIDOCTOR.RU 